\title{
Association of mast cell infiltration with gastric cancer progression
}

\author{
BEI ZHONG ${ }^{1}, \mathrm{YU} \mathrm{LI}^{2}, \mathrm{XIAODONG} \mathrm{LIU}^{2}$ and DONGSHENG WANG ${ }^{2}$ \\ Departments of ${ }^{1}$ Hyperbaric Oxygen and ${ }^{2}$ General Surgery, The Affiliated Hospital \\ of Qingdao University, Qingdao, Shandong 266003, P.R. China
}

Received May 14, 2016; Accepted July 27, 2017

DOI: $10.3892 / 01.2017 .7380$

\begin{abstract}
The present study aimed to determine the expression of mast cells, C-C motif chemokine ligand 2 (CCL-2) and C-C motif chemokine receptor 2 (CCR2) in gastric cancer tumor tissue; and the association of mast cells with the proliferation, migration, invasion and apoptosis of gastric cancer cells. In addition, whether the stem cell factor (SCF)/c-Kit pathway was associated with the secretion of CCL-2 by gastric cancer cells was explored. Flow cytometry analysis and immunohistochemistry were used to observe the relative number of mast cells, and reverse transcription-quantitative polymerase chain reaction and western blot analysis were utilized to determine the expression of CCL-2 and CCR2 mRNA and protein. Following the co-culture of the mast cell line HMC-1 and the gastric cancer cell line BGC-823, a Transwell assay was used to validate the effect of mast cells on the migration and invasion of gastric cancer cells. Furthermore, Cell Counting kit-8 and dual acridine orange/ethidium bromide fluorescent staining assays were performed to determine the proliferation and apoptosis of gastric cancer cells, following co-culture with mast cells. The expression of SCF and c-Kit were also determined with a western blot analysis. A specific phosphoinositide 3-kinase (PI3K) inhibitor, wortmannin, was used to test the effect of PI3K inhibition on the secretion of CCL-2 in gastric cancer. The results demonstrated that the proportion of infiltrating mast cells, and the mRNA/protein expression of CCL-2 and CCR2, were significantly increased in tumor tissue relative to adjacent tissues. In addition, the migration and invasion of gastric cancer cells were significantly increased when mast cells were used as an attractant. When co-cultured with mast cells, the viability of gastric cancer cells was significantly increased and $\mathrm{H}_{2} \mathrm{O}_{2}$-induced apoptosis was inhibited. In gastric cancer tissue samples, the expression of SCF, c-Kit and phosphorylated (p)-Akt protein were significantly increased compared with normal adjacent tissues. It was hypothesized
\end{abstract}

Correspondence to: Dr Dongsheng Wang, Department of General Surgery, The Affiliated Hospital of Qingdao University, 16 Jiangsu Road, Qingdao, Shandong 266003, P.R. China

E-mail: dongsheng0509@sina.com

Key words: gastric cancer, mast cell, tumor cells, migration, invasion that SCF/c-Kit signaling pathway was activated by PI3K-Akt, resulting in an increase in the expression of CCL-2 mRNA and protein. Furthermore, it was demonstrated that CCL-2 mRNA and protein expression was significantly inhibited by treatment with the PI3K inhibitor wortmannin. Additionally, wortmannin intervention significantly inhibited gastric cancer cell migration and invasion. Therefore, the results of the present study demonstrated that mast cells may promote gastric cancer cell proliferation, migration and invasion, and inhibit apoptosis. In addition, the activation of the SCF/c-Kit signaling pathway was identified to promote the expression of CCL-2, which is associated with the development and metastasis of gastric cancer.

\section{Introduction}

Gastric cancer is one of the most common types of malignant tumor in clinical practice and is a leading cause for mortality (1). Gastric cancer causes the second highest number of mortalities of all types of malignant tumor (2). The majority of tumor types, particularly solid tumors, are associated with infection and chronic inflammation (3). The occurrence and development of gastric cancer are influenced by interactions with cells in the tumor microenvironment, and it is particularly associated with chronic inflammation (4). In gastric tumors there is a combination of gastric cancer cells and a range of types of immune cell, which may either inherently exist in the tumor or migrate from other tissues $(4,5)$. Immune cells in the tumor microenvironment may alter the phenotype of gastric cancer cells and regulate the inflammatory response through a number of signaling pathways, thereby promoting the development of gastric cancer (6).

Myeloid cells are the principal type of immunosuppressive cell in the tumor microenvironment (7). Mast cells are a type of terminally differentiated myeloid cell and serve functions in type I allergic reactions, innate and acquired immunity by identifying and eliminating pathogens, releasing bio-active factors, and preventing invasion by pathogens (8). Previous studies have identified that the extent of mast cell infiltration was enhanced in a number of types of tumor tissue, including breast cancer and lymphoma, suggesting that mast cells serve a function in the development of tumors $(9,10)$. However, a limited number of studies have been conducted on mast cells in gastric cancer. The effects of mast cells on the occurrence and development of gastric cancer, and the mechanism of inflammatory regulation, remain unknown. 
Chemokines are small, soluble proteins that induce the directional chemotaxis of cells by forming a concentration gradient (11). C-C motif chemokine ligand 2 (CCL-2) is a member of the $\mathrm{CC}$ chemokine subfamily that may be secreted by monocytes, epithelial cells, astrocytes or tumor cells, and is a key molecule for the regulation of inflammation (12). C-C chemokine receptor 2 (CCR2) is a member of the $\mathrm{G}$ protein-coupled receptors superfamily and binds CCL-2 with high affinity. Previous studies indicated that CCL-2 or CCR2 are highly expressed in a number of types of tumor tissue (13), and that a CCL-2 recombinant may induce the malignant transformation of breast epithelial cells (14). In addition, it was identified that the increased expression of CCL-2 in gastric cancer tissue and peripheral blood was associated with disease progression (15); however, whether CCL-2 and CCR2 are involved in the development of gastric cancer, and the underlying molecular mechanism of this interaction, remains unknown.

Stem cell factors (SCFs), also known as mast cell growth factors, may regulate the growth of a number of types of cell, induce the proliferation and survival of and mobilize stem/progenitor cells, and regulate the development of mast cells $(16,17)$. Mast/stem cell growth factor receptor (known as c-Kit or CD117) is a transmembrane protein with tyrosine kinase activity that acts as a receptor for SCFs; the activation of c-Kit is associated with the proliferation and differentiation of cells (18). It has been demonstrated that a mutation of the c-Kit gene may cause the deregulation of the proliferation and apoptosis of cells, promoting the development of malignant tumors (19). c-Kit has been identified as highly expressed in lung cancer, gastrointestinal stromal tumors, ovarian cancer and other types of malignant tumor (19). However, the association between the activation of the $\mathrm{SCF} / \mathrm{c}$-kit signaling pathway and the chemotaxis of mast cells, as induced by the secretion of CCL-2, in gastric cancer tissue is yet to be reported.

\section{Materials and methods}

Patient tissues. Cancer tissues and adjacent-normal tissue were collected from 40 patients who underwent gastrectomy for gastric cancer in the Affiliated Hospital of Qingdao University (Qingdao, China) between January 2011 and December 2013. Of the 40 specimens, 26 were from male patients and 14 from female patients, with an age range of $42-76$ years, and a mean age of $56.25 \pm 11.37$ years. All patients provided written, informed consent. Fresh tissue specimens were pathologically confirmed and diagnosed; differentiation and stage were determined as previously described (20). Of the specimens, 7, 10 and 23 cases were highly, moderately and poorly differentiated, respectively, and 5, 9, 10, and 16 cases were classified as stage I, II, III and IV, respectively.

Experimental materials. The human mast cell line HMC-1 was purchased from Shanghai Meiyan Biological Technology Co., Ltd. (Shanghai, China). HMC-1 cells were cultured in RPMI 1640 medium containing 10\% fetal bovine serum and $1 \%$ penicillin/streptomycin (all Gibco; Thermo Fisher Scientific, Inc., Waltham, MA, USA) and incubated at $37^{\circ} \mathrm{C}$ with $5 \% \mathrm{CO}_{2}$ in air. The human gastric cancer cell line BGC-823 was purchased from Shenzhen Baienwei Biological Technology Co., Ltd, Shanghai, China. BGC-823 cells were maintained in RPMI-1640 medium supplemented with $10 \%$ heated-inactivated calf serum (Hangzhou Sijiqing Biological Engineering Materials Co., Ltd., Hangzhou, China) and 1\% penicillin/streptomycin at $37^{\circ} \mathrm{C}$ with $5 \% \mathrm{CO}_{2}$ in air.

The reagents for flow cytometry, included a phycoerythrin (PE-) cyanine (Cy) 7-labeled anti-human cluster of differentiation (CD) 45 antibody (560915, BD Biosciences, San Jose, CA, USA), a peridinin chlorophyll protein complex (PerCP-) Cy5.5-labeled anti-CD117 antibody (cat. no. 560557; BD Biosciences), a fluorescein isothiocyanate (FITC)-labeled anti-high-affinity immunoglobulin E receptor (FceRI $\alpha$ ) antibody (cat. no. 610167; BD Biosciences), an allophycocyanin (APC)-labeled anti-major histocompatibility complex class II-DR (HLA-DR) antibody (cat. no. 559868; BD Biosciences).

RPMI 1640 and collagenase type IV were purchased from Gibco; Thermo Fisher Scientific, Inc., and DNase type I was purchased from Sigma-Aldrich; Merck KGaA (Darmstadt, Germany). An immunohistochemical staining kit was purchased from Wuhan BOSHIDE Biological Engineering Co., Ltd. (Wuhan, China) and the EnVision ${ }^{\mathrm{TM}}$ Gl2 System/AP kit was purchased from Dako; Agilent Technologies, Inc. (Santa Clara, CA, USA).

The mouse anti-CCL-2 (cat. no. sc-1304) and anti-CCR2 (cat. no. sc-6228) antibodies were purchased from Cell Signaling Technology, Inc. (Danvers, MA, USA) and an anti-GAPDH (cat. no. sc-365062) antibody was purchased from Santa Cruz Biotechnology, Inc. (Dallas, TX, USA). The rabbit anti-SCF (cat. no. sc-9132), and anti-c-KIT (cat. no. sc-168) antibodies were purchased from Santa Cruz Biotechnology, Inc., and anti-Akt (cat. no. 9272) and anti-phosphorylated Akt (p-Akt; cat. no. 9271S) were purchased from Cell Signaling Technology, Inc.

The reagents for reverse transcription-quantitative polymerase chain reaction (RT-qPCR), including the RNA extraction reagent, the RT kit and the SYBR kit, were purchased from Takara Biological Co., Ltd. (Dalian, China). The primers for CCL-2, CCR2 and GAPDH, as described in Table I, were purchased from Shanghai Yingjun Biotechnology Co. Ltd (Shanghai, China).

Sample processing. The fresh specimens from patients with gastric cancer, including the gastric cancer and normal adjacent tissues $(0-5 \mathrm{~cm}$ from the margin of the gastric cancer tissue), were processed under aseptic conditions. Following 2-3 washes with $0.01 \mathrm{~mol} / \mathrm{l} \mathrm{PBS}$, tissues were cut into small pieces and transferred into tissue lysis buffer. The formula of the buffer was as follows: Collagenase type IV (final concentration, $1 \mathrm{mg} / \mathrm{ml}$ ), DNase type I (final concentration, $100 \mu \mathrm{g} / \mathrm{ml}$ ), $\mathrm{CaCl}_{2}$ (final concentration, $2 \mathrm{mmol} / \mathrm{l}$ ) and $\mathrm{MgCl}_{2}$ (final concentration, $2 \mathrm{mmol} / \mathrm{l}$ ). The tissues were isolated using an automatic single cell separator and incubated for $60 \mathrm{~min}$ at $37^{\circ} \mathrm{C}$, with continuous rotation. Subsequently, the samples were ground, passed through a 200-mesh steel net and centrifuged at $500 \mathrm{x} g$ for $5 \mathrm{~min}$ at the room temperature. Finally, the supernatant was discarded and the single cell suspension was collected for staining and flow cytometry.

Flow cytometry staining. The single cell suspensions isolated from gastric cancer and adjacent tissues were labeled using the PE-Cy7-labeled anti-CD45 (1:600), FITC-labeled antiFceRIa (1:100), PerCP-Cy5.5-labeled anti-CD117 (1:500), 
Table I. Primer sequences of the target genes.

\begin{tabular}{lll}
\hline Gene & \multicolumn{1}{c}{ Forward primer } & \multicolumn{1}{c}{ Reverse primer } \\
\hline CCL-2 & GCTCATAGCAGCCACCTTCATTC & CCGCCAAAATAACCGATGTGATAC \\
CCR2 & CCAACTCCTGCCTCCGCTCTA & TGCAGATTCTTGGGTTGTGGAG \\
GAPDH & ACCACAGTCCATGCCATCAC & TCCACCACCCTGTTGCTGTA
\end{tabular}

Sequences are in the 5' to 3' direction. CCL-2, C-C motif chemokine ligand 2; CCR2, C-C motif chemokine receptor 2.

APC-labeled anti-HLA-DR (1:300) antibodies at $4^{\circ} \mathrm{C}$ for $30 \mathrm{~min}$. PBS containing $1 \%$ bovine serum albumin $(100 \mu \mathrm{l}$, Sigma-Aldrich; Merck KGaA) was used to wash the samples. The mixtures were subsequently centrifuged at $500 \mathrm{x} \mathrm{g}$ for $5 \mathrm{~min}$ at $4^{\circ} \mathrm{C}$ and fixed with $4 \%$ polyformaldehyde at $4^{\circ} \mathrm{C}$ for $30 \mathrm{~min}$. Following a further wash in PBS, the precipitant was collected by centrifuging at $500 \mathrm{x}$ g for $5 \mathrm{~min}$ at $4^{\circ} \mathrm{C}$, and $500 \mu \mathrm{l}$ PBS was added to re-suspend the cells. The mixture was incubated at $4^{\circ} \mathrm{C}$ in lightproof conditions prior to flow cytometry to detect the $\mathrm{CD} 45^{+}$and $\mathrm{CD} 117^{+} \mathrm{Fc} \mathrm{RI} \alpha^{+}$cell subpopulations. FlowJo software version 7.6.2 (FlowJo LLC, Ashland, OR, USA) was used for data processing.

Immunohistochemistry. Gastric cancer and adjacent tissue samples were fixed in $10 \%$ formalin for $12 \mathrm{~h}$ at $4^{\circ} \mathrm{C}$, embedded in paraffin and sliced into $4-\mu \mathrm{m}$ sections. The sections were dewaxed using xylene twice (each for $10 \mathrm{~min}$ ), rehydrated using an $80 \%$ ethanol series for $1 \mathrm{~min}$ and washed in distilled water. Antigen retrieval was performed by immersing slides in ethylenediaminetetraacetic acid for $15 \mathrm{~min}$ at $95^{\circ} \mathrm{C}$, and the sections were allowed to cooled at room temperature. Subsequently, sections were incubated with tryptase (dilution, 1:100) and a primary antibody (anti-Akt, 1:1,000) for $30 \mathrm{~min}$ at room temperature and washed with PBS three times (each for 5 min). The IHC staining was developed with the EnVision ${ }^{\mathrm{TM}}$ Gl2 System/AP kit, according to the manufacturer's protocol.

RT-qPCR detection. The gastric cancer tissue samples or BGC-823 cells were washed with PBS and $1 \mathrm{ml}$ TRIzol reagent (Invitrogen; Thermo Fisher Scientific, Inc.) was added. The tissue samples were ground. Subsequently, total RNA was extracted using the phenol chloroform extraction method, and the concentration and purity of tissue RNA were determined using ultraviolet spectrophotometry. RNA was reverse transcribed into cDNA using the RT kit according to the manufacturer's protocol (Takara Biotechnology Co., Ltd., Dalian, China). The expression of CCL-2, CCR2 and GAPDH mRNA was determined using the SYBR qPCR kit with the primers listed in Table I. qPCR was performed using the Applied Biosystems StepOne Real-Time PCR instrument (Thermo Fisher Scientific, Inc.), and $\mathrm{Cq}$ values were automatically calculated with the in-built software. The qPCR conditions were as follows: Pre-denaturation at $95^{\circ} \mathrm{C}$ for $20 \mathrm{sec}$, then $95^{\circ} \mathrm{C}$ for $5 \mathrm{sec}$ and $60^{\circ} \mathrm{C}$ for $30 \mathrm{sec}$ for 45 cycles. GAPDH was used as an internal reference gene.

Western blot analysis. The gastric cancer and adjacent tissues were cut into pieces, and the BGC-823 gastric cancer cells and tissue samples were lysed with the aforementioned tissue lysis buffer (Sample processing). The lysis solutions were centrifuged at $500 \mathrm{x} \mathrm{g}$ at $4^{\circ} \mathrm{C}$ for $15 \mathrm{~min}$ and the total protein in the supernatant was extracted and cryopreserved at $-80^{\circ} \mathrm{C}$. The protein concentration was determined using the bicinchoninic acid method. A total of $30 \mu \mathrm{g}$ protein per lane was separated using SDS-PAGE electrophoresis and the products were transferred to a PVDF membrane with a $350 \mathrm{~mA}$ current. The film was blocked with $5 \%$ skimmed milk powder for $1 \mathrm{~h}$ and incubated with the following primary antibodies at $4^{\circ} \mathrm{C}$ overnight: Anti-SCF (1:200), c-Kit (1:500), Akt (1:1,000), p-Akt (1:1,000), CCL-2 (dilution, 1:1,000), CCR2 $(1: 1,000)$ and GAPDH $(1: 2,000)$. The membrane was rinsed with Tris buffered saline with Tween-20 (TBST) three times, for $10 \mathrm{~min}$ each time, and the corresponding horseradish peroxidase-conjugated goat anti-rabbit secondary antibody (cat. no. ab6721; dilution, 1:5,000, Abcam, Cambridge, UK) was added. Following incubation at room temperature for $2 \mathrm{~h}$, the film was washed with TBST (each time for $10 \mathrm{~min}$ ), and the target protein was developed using an enhanced chemiluminescence reagent (Thermo Fisher Scientific, Inc.), and the relative density of the bands were analyzed using ImageLab software version 4.1 (Bio-Rad Laboratories Inc., Hercules, MA, USA). Using GAPDH as an internal reference, the relative expression of CCL-2 and CCR2 proteins were calculated.

Transwell migration and invasion assay. Prior to the migration assay, $600 \mu \mathrm{l}$ blank control (PBS) and HMC-1 mast cells $\left(5 \times 10^{4}\right.$ cells $\left./ \mathrm{ml}\right)$ were added to the lower chambers of 24 -well plates, with triplicates for each well. BGC-823 cell suspensions ( $200 \mu \mathrm{l}$, density, $2 \times 10^{4}$ cells $\left./ \mathrm{ml}\right)$ were seeded in the upper chamber of Transwell inserts and incubated for $24 \mathrm{~h}$. The plate was washed with distilled water, allowed to dry, fixed with $70 \%$ paraformaldehyde for $15 \mathrm{~min}$ and stained with $0.05 \%$ crystal violet (Bioteke Corporation, Beijing, China) for $30 \mathrm{~min}$. Each plate was viewed with an optical microscope at X100 magnification in five fields of view (upper, lower, left, right and center) and the numbers of migrating cells were counted. The assay was repeated 3 times.

The invasion assay was similar to the migration assay, except $12 \mathrm{~h}$ prior to the addition of cells, RPMI 1640 was mixed with Matrigel, at a ratio of $3: 1$ at $4^{\circ} \mathrm{C}$, and $40 \mu 1$ of the mixture was added into the upper Transwell chambers. The density of the inoculated gastric cancer cells was $4 \times 10^{4}$ cells $/ \mathrm{ml}$ for the invasion assay.

Cell Counting kit (CCK)-8 proliferation assay. BGC-823 cells in the logarithmic growth stage were inoculated in 
96-well plates at a density of $5 \times 10^{3}$ cells/well and incubated at $37^{\circ} \mathrm{C}$ with $5 \% \mathrm{CO}_{2}$. Once the cells had adhered, the old culture medium was discarded and replaced with fresh RPMI 1640. HMC-1 mast cells were added to a concentration of 105 cells $/ \mathrm{ml}$. The negative control group, without HMC-1 cells, was also seeded. A total of $300 \mathrm{nM}$ Wortmannin (LC Laboratory, Woburn, MA, USA) was added into 96-well plates $1 \mathrm{~h}$ before the CCK- 8 assay. A CCK- 8 assay was performed at 12 and $24 \mathrm{~h}$ post-incubation; $10 \mu \mathrm{l}$ CCK- 8 solution was added into each well and incubated for $2 \mathrm{~h}$. The absorbance value was measured at $490 \mathrm{~nm}$ using a microplate reader. The assay was repeated three times, in triplicate.

Acridine orange (AO)/ethidium bromide (EB) double fluorescent staining apoptosis. Following co-cultivation, BGC-823 cells were seeded at a density of $5 \times 10^{4}$ cells/well and HMC-1 cells were seeded at a density of $2 \times 10^{4}$ cells/well in 24-well plates for $24 \mathrm{~h}$, and apoptosis was induced by adding $100 \mu \mathrm{M} \mathrm{H}_{2} \mathrm{O}_{2}$ for $12 \mathrm{~h}$. Subsequent to washing in PBS, the cells were fixed with $4 \%$ paraformaldehyde at room temperature for $10 \mathrm{~min}$. PBS was added and agitated two times (each for $3 \mathrm{~min})$. AO solution was mixed with EB solution at a ratio of 1:1. AO/EB mixture $(10 \mu \mathrm{l})$ was removed and combined with $90 \mu \mathrm{l}$ Buffer A (Abcam). The $100 \mu 1$ mixture was added into each well, incubated for $5 \mathrm{~min}$ at room temperature, washed with PBS twice for $3 \mathrm{~min}$, and allowed to dry in light proof conditions. Anti-fluorescence quenching mounting media was added prior to observing changes to cell morphology and nuclear structures using an Olympus IX50 inverted fluorescence microscope (Olympus Corporation, Tokyo, Japan) at x400 magnification. Normal cells exhibited uniform green fluorescence, whereas chromatin condensation and nuclear dissolution were visible in the apoptotic cells, and compact, hyperchromatic yellow green particles or buds in different sizes could be observed. This assay was repeated 3 times.

Statistical analysis. The data were analyzed using SPSS software (version 19.0; IBM Corp., Armonk, NY, USA) and were presented as the mean \pm standard deviation. Comparisons between two groups were performed using a t-test and comparisons between three groups were examined by one-way analysis of variance followed by a Dunnett's post hoc test. $\mathrm{P}<0.05$ was considered to indicate a statistically significant difference.

\section{Results}

Mast cells are upregulated in tumor tissue compared with adjacent tissues. Single cell suspensions were prepared from the surgical tissue specimens of 40 cases of patients with gastric cancer. The infiltration ratio of mast cells in the gastric cancer tissues and para-cancerous tissues was analyzed by calculating the ratio of $\mathrm{CD} 117^{+} \mathrm{Fc} \varepsilon \mathrm{RI} \alpha^{+}$mast cells to $\mathrm{CD} 45^{+}$cells. The results of flow cytometry demonstrated that the proportion of mast cells in gastric cancer tissue $(14.73 \pm 4.62 \%)$ was significantly increased compared with the adjacent tissues $(10.03 \pm 3.02 \%$; Fig. $1 \mathrm{~A}$ and $\mathrm{B} ; \mathrm{P}<0.001)$.

Immunohistochemical staining was performed to stain mast cells red with tryptase in gastric cancer and adjacent tissues. The results of immunohistochemistry revealed that the number of mast cells in gastric cancer tissue was increased, compared with that in the adjacent cancer tissue (Fig. 1C). The results of flow cytometry and immunohistochemistry indicated that gastric cancer tissue was enriched in mast cells, which may serve a function in the development of gastric cancer.

$C C L-2$ and CCR2 are upregulated in tumor tissue compared with adjacent tissues. RT-qPCR revealed that the expression of CCL-2 and CCR2 mRNA was significantly increased in gastric cancer tissues compared with that in the adjacent tissues $(\mathrm{P}<0.001$; Fig. 2A and B). The results of western blot analysis demonstrated that the expression of CCL-2 and CCR2 protein was significantly increased in gastric cancer tissues compared with adjacent tissues (Fig. 2C and D; P<0.001). RT-qPCR and western blot analysis suggested that the expression levels of chemokine CCL-2 and its receptor CCR2 in gastric cancer tissues were increased compared with the adjacent tissues.

Mast cells significantly enhance the migratory and invasive abilities of gastric cancer cells. In the migration assay, BGC-823 gastric cancer cells in the control group demonstrated a limited capacity for migration, with a mean of $2.15 \pm 1.36$ cells per well migrating to the lower surface of the membrane. However, when HMC-1 mast cells were added to the lower chamber, the migratory ability of BGC-823 cells was enhanced, with a mean of $21.68 \pm 4.29$ cells per well migrating to the lower surface (Fig. 3A and B; P<0.01).

Similar to the migration assay result, $2.01 \pm 1.30$ BGC-823 cells per well invaded to the lower surface of the membrane. However, when HMC-1 mast cells were added to the lower chamber, the invasive ability of gastric cancer cells was significantly enhanced, with $52.73 \pm 12.08$ cells invading to the lower surface of the membrane (Fig. $3 \mathrm{C}$ and $\mathrm{D} ; \mathrm{P}<0.001$ ). Transwell migration and invasion assays revealed that mast cells may significantly enhance the migratory and invasive abilities of gastric cancer cells.

Co-culturing with mast cells promotes the proliferation and inhibits the apoptosis of gastric cancer cells. As determined with a CCK-8 assay, the OD values for BGC-823 cells with or without HMC-1 cells increased; however, the OD value determined in the co-culture group was significantly increased compared with the control group at 12 and $24 \mathrm{~h}$ (Fig. 4A; $\mathrm{P}<0.05$ ). Prior to the induction of apoptosis by $\mathrm{H}_{2} \mathrm{O}_{2}$, the apoptosis rates were $6.50 \pm 1.25$ (co-culture group) and $6.93 \pm 1.55 \%$ (control group), which was not significantly different. Following co-culture with HMC-1 cells for $24 \mathrm{~h}$, the apoptosis rate of gastric cancer cells was significantly decreased $(26.87 \pm 4.68 \%)$ compared with the control group (42.63 $\pm 5.89 \%$; Fig. $4 \mathrm{~B} ; \mathrm{P}<0.01)$. Prior to $\mathrm{H}_{2} \mathrm{O}_{2}$ treatment, the majority of cells demonstrated a uniform green fluorescence with a limited amount of yellow fluorescence. Following $\mathrm{H}_{2} \mathrm{O}_{2}$ treatment for $12 \mathrm{~h}$, the number of cells with incomplete nuclear membranes increased, dense patches could be observed in the membrane, and orange or yellow apoptotic bodies were observed (Fig. 4C). Following the co-culture with mast cells, a reduced number of pale yellow gastric cancer cells and apoptotic bodies were observed (Fig. 4C). The results of AO/EB double fluorescence staining demonstrated that co-culturing with mast cells may promote the proliferation, and inhibit the apoptosis, of gastric cancer cells. 
A

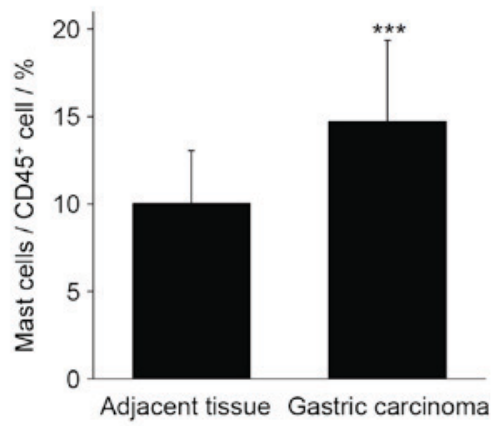

C

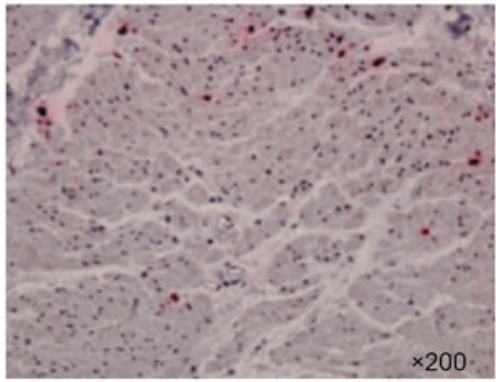

Adjacent tissue
B
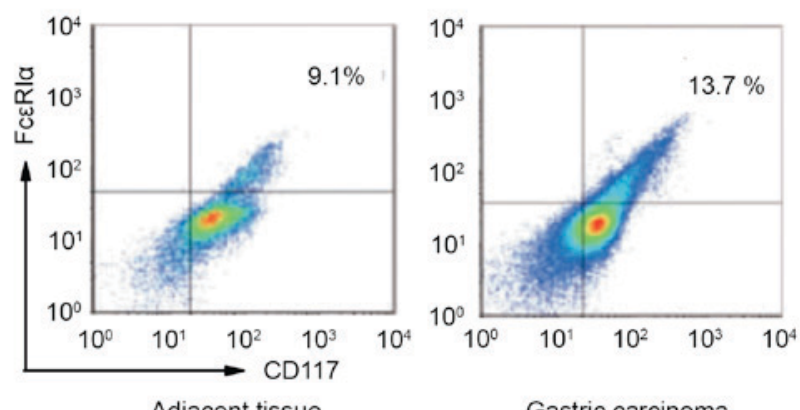

Gastric carcinoma

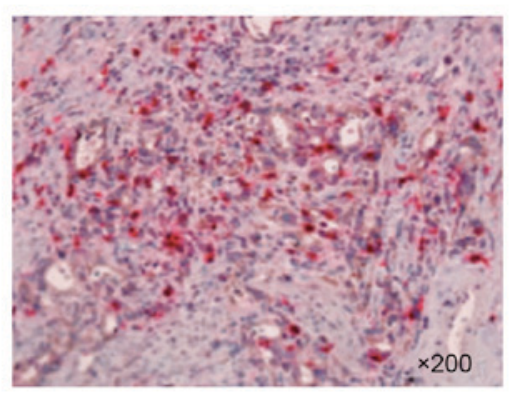

Gastric carcinoma

Figure 1. Differential expression of mast cells in gastric cancer and adjacent tissues. (A) The proportion of mast cells was significantly increased in gastric cancer, compared with adjacent tissue. (B) Flow cytometry and (C) immunohistochemical analysis validated that the expression of mast cells in gastric cancer tissues was increased, compared with adjacent tissue mast cells (x200 magnification). Representative images are presented. ${ }^{* * *} \mathrm{P}<0.001$ vs. adjacent tissue. $\mathrm{CD}$, cluster of differentiation; FceRI $\alpha$, high-affinity immunoglobulin E receptor.

A

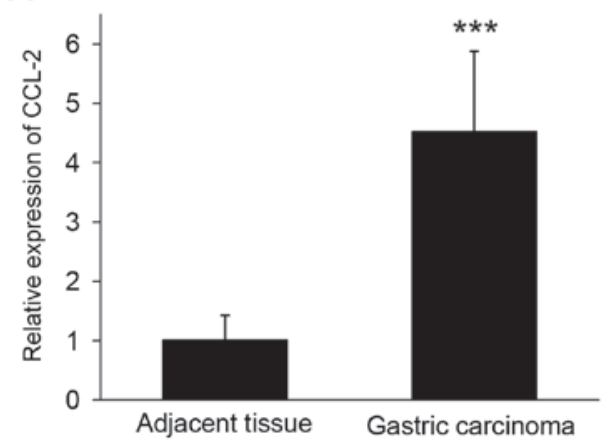

C

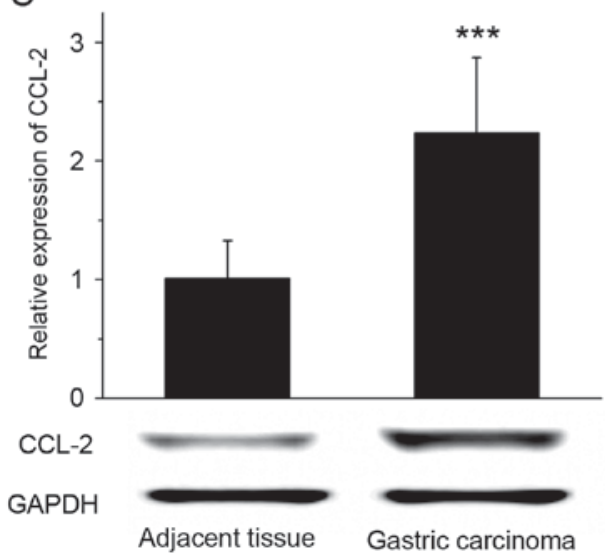

B

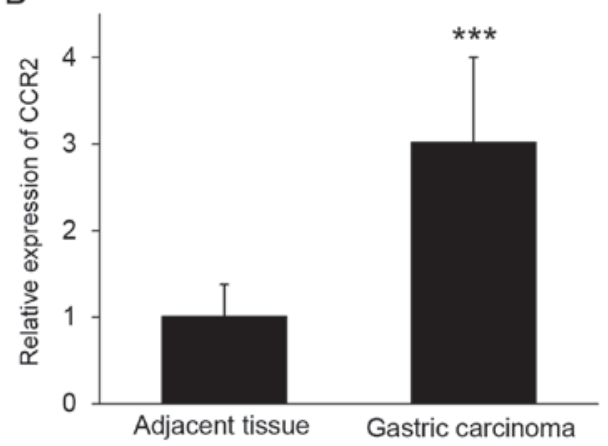

D

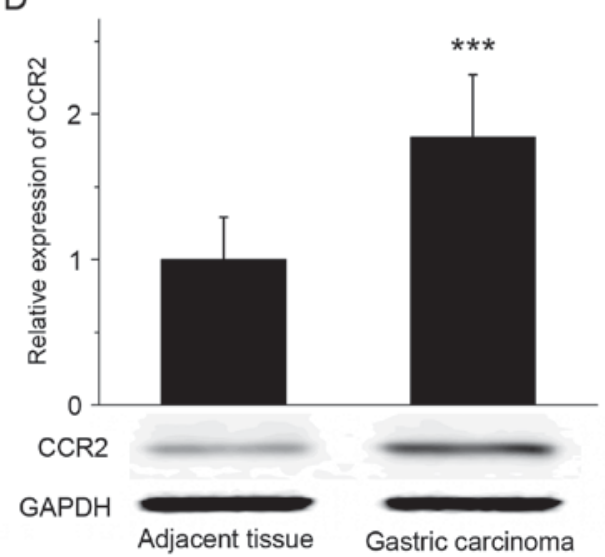

Figure 2. Differential expression of CCL-2 and CCR2 in gastric cancer and adjacent tissues. The reverse transcription-quantitative polymerase chain reaction was used to determine that the relative expression of (A) CCL-2 and (B) CCR2 mRNA was increased in gastric cancer tissues compared with adjacent tissues. Western blot analysis demonstrated that the expression level of the (C) CCL-2 and (D) CCR2 proteins were significantly increased in gastric cancer tissues compared with adjacent tissues. GAPDH was used as the loading control. ${ }^{* * *} \mathrm{P}<0.001$ vs. adjacent tissues. CCL-2, C-C motif chemokine ligand 2; CCR2, C-C motif chemokine receptor 2 . 


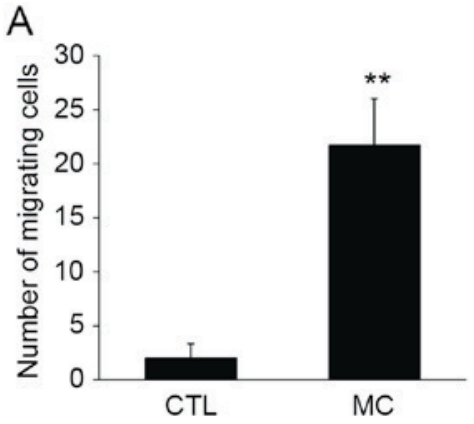

C

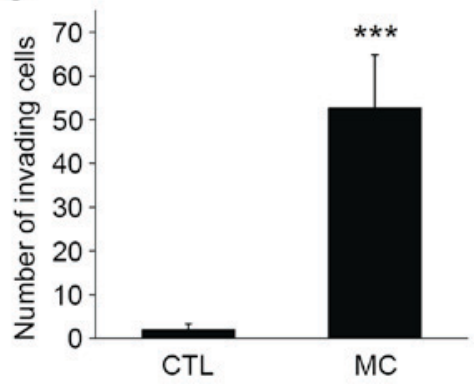

B

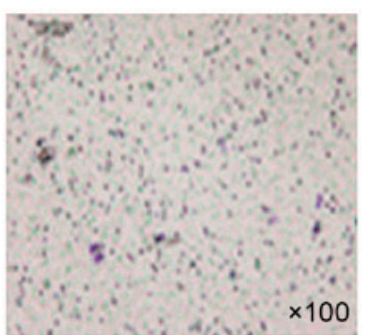

CTL

$\mathrm{D}$

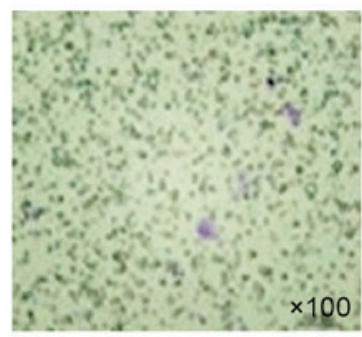

CTL

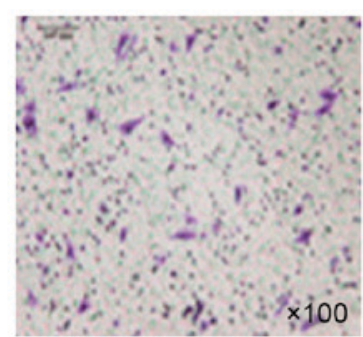

$\mathrm{MC}$



$\mathrm{MC}$

Figure 3. Effect of mast cells on gastric cancer cell invasion and migration. (A) Co-culture of mast cells with gastric cell cancer increased the migratory abilities of gastric cancer cells compared with the CTL. (B) Image of the migration assay (magnification, x100). (C) Following co-culture with mast cells, the invasive abilities of gastric cancer cells significantly increased compared with the CTL. (D) Image of the invasion assay (magnification, $\mathrm{x} 100$ ). ${ }^{* *} \mathrm{P}<0.01$ and ${ }^{* * * *} \mathrm{P}<0.001$ vs. CTL. CTL, control group; MC, mast cell co-culture group.

A
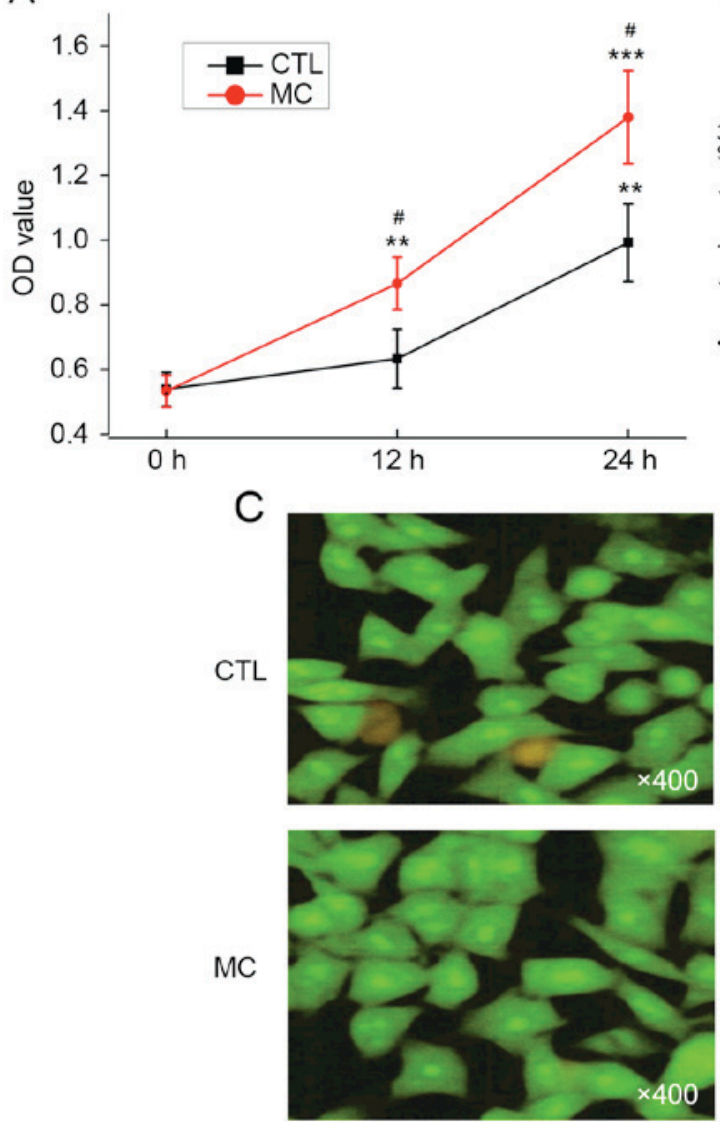

$0 \mathrm{~h}$
B
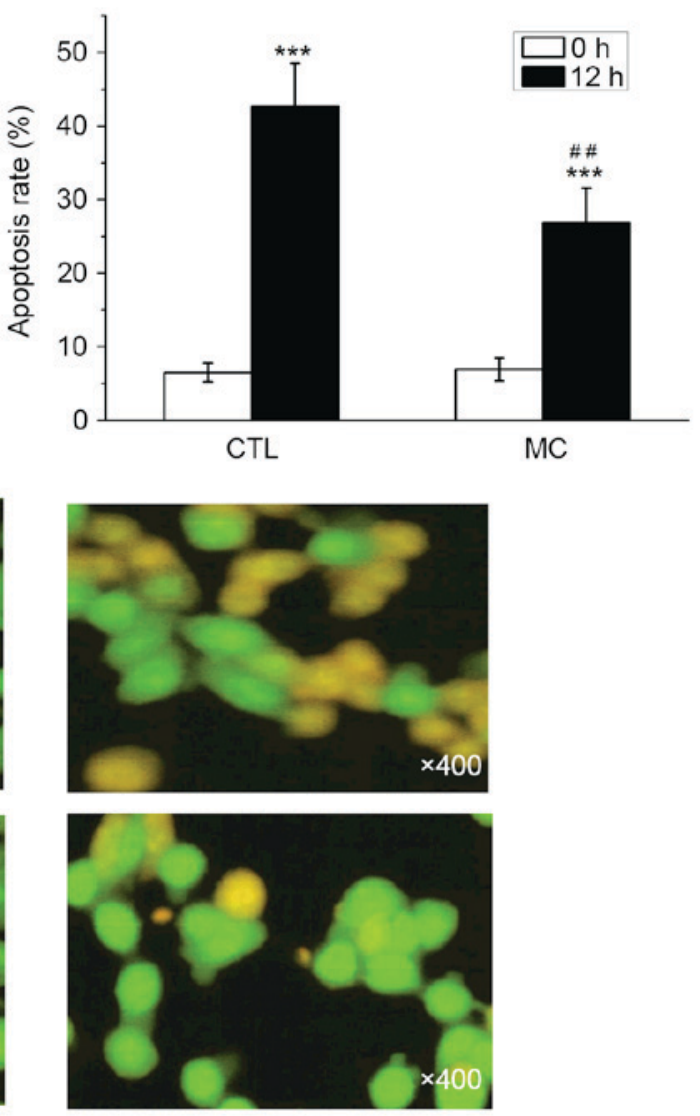

$12 \mathrm{~h}$

Figure 4. Effect of mast cells on gastric cancer cell proliferation and apoptosis. (A) Following a Cell Counting kit-8 assay, the OD value determined in the co-culture group (mast cells with gastric cancer cells) was significantly increased compared with the CTL. (B) Following co-culture, the apoptosis rate of gastric cancer cells significantly decreased, compared with the control group. (C) Representative images of dual-fluorescence staining. ${ }^{* *} \mathrm{P}<0.01$ and ${ }^{* * * *} \mathrm{P}<0.001$ vs. $0 \mathrm{~h} ;{ }^{\# \mathrm{P}}<0.05$ and ${ }^{\# \#} \mathrm{P}<0.01$ vs. CTL. OD, optical density; CTL, control group; MC, mast cell co-culture group. 
A


C

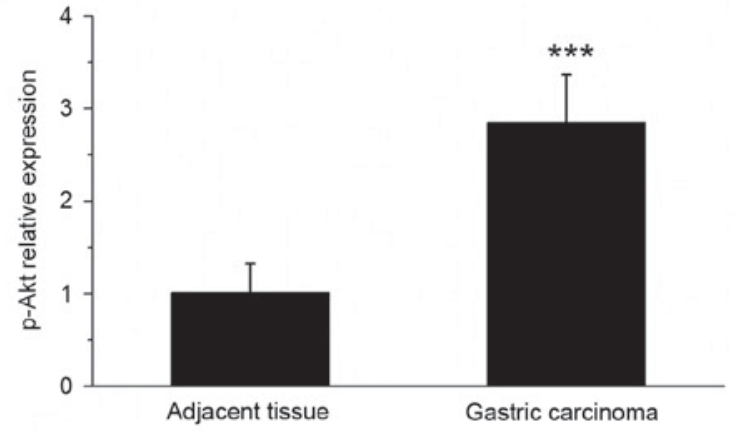

p-Akt

GAPDH
B

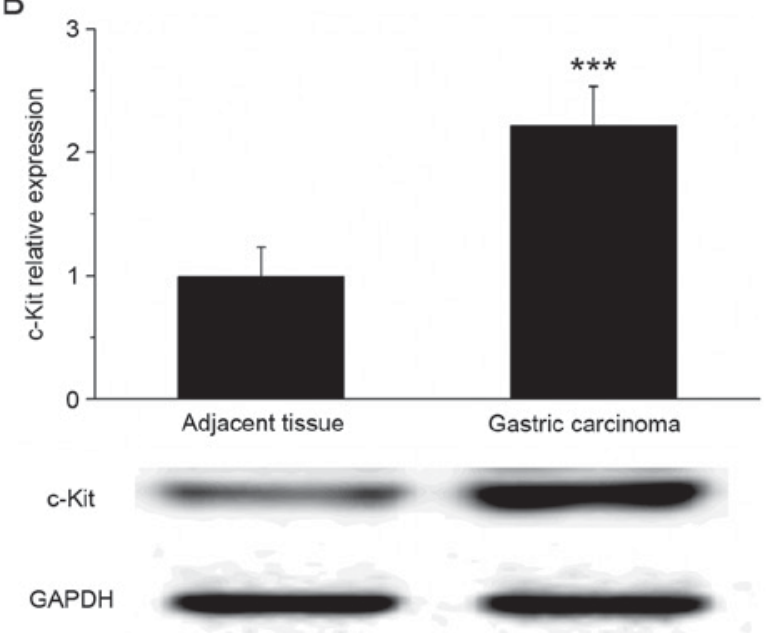

D

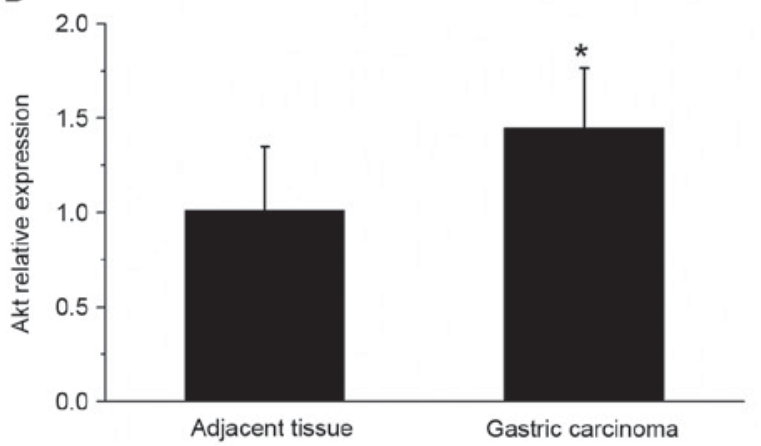

Akt

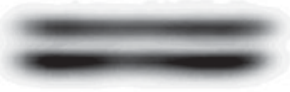

GAPDH

Figure 5. Differential expression of SCF/c-Kit in gastric cancer and adjacent tissues. Western blot analysis revealed that the expression levels of (A) SCF, (B) c-Kit, (C) p-Akt and (D) Akt were significantly increased in gastric cancer tissues, compared with adjacent tissues. GADPH was used as the loading control. ${ }^{*} \mathrm{P}<0.05,{ }^{* *} \mathrm{P}<0.01$ and ${ }^{* * *} \mathrm{P}<0.001$ vs. adjacent tissues. $\mathrm{SCF}$, stem cell factor; $\mathrm{p}-$, phosphorylated.

SCF and c-Kit are upregulated in gastric cancer tissue. The results of western blot analysis revealed that the expression of SCF and c-Kit protein was significantly increased in gastric cancer tissues, compared with that in the adjacent tissues (Fig. 5A and $\mathrm{B} ; \mathrm{P}<0.01$ ). In addition, the expression of $\mathrm{p}$-Akt in gastric cancer tissue was significantly increased compared with that in the adjacent tissues $(\mathrm{P}<0.001)$; however, the difference in the Akt expression level of the two types of tissue was relatively small (Fig. 5C and D; $\mathrm{P}<0.05$ ). These results suggested that the $\mathrm{SCF} / \mathrm{c}-\mathrm{K}$ it signaling pathway may activate the phosphoinositide 3-kinase (PI3K)-Akt signaling pathway to participate in the development and metastasis of gastric cancer.

Pre-treatment with wortmannin inhibits the expression of CCL-2 and reduces the migratory abilities of gastric cancer cells. RT-qPCR and western blot analysis revealed that CCL-2 expression was increased in BGC-823 cells co-cultured with HMC-1 cells; however, if cells were pre-treated with the specific $\mathrm{PI} 3 \mathrm{~K}$ inhibitor wortmannin, the mRNA and protein expression of CCL-2 was significantly inhibited (Fig. 6A and $\mathrm{B} ; \mathrm{P}<0.05)$.
In the migration assay, when $\mathrm{HMC}-1$ cells were added to the lower chamber, the number of BGC-823 cells on the lower surface of the membrane was $19.84 \pm 4.52$; however, the number decreased to $8.22 \pm 2.49(\mathrm{P}<0.01)$ following wortmannin treatment (Fig. 6C). In the invasion assay, when HMC-1 cells were added to the lower chamber, the number of BGC-823 cells on the lower surface of the membrane was $44.73 \pm 10.09$; however, this decreased to $20.73 \pm 5.21(\mathrm{P}<0.001)$ following wortmannin treatment (Fig. 6D). These results suggested that the PI3K inhibitor may have inhibited the expression of CCL-2 in gastric cancer cells, and the inhibitor may inhibit the migration and invasion of gastric cancer cells promoted by mast cells.

\section{Discussion}

Gastric cancer is one of the most common types of cancer and the leading causes of cancer-associated mortalities in china (21). Despite a sustained period of significant progress in basic and clinical cancer research, the current status of the diagnosis and treatment of gastric cancer is poor; the majority of patients with gastric cancer are diagnosed at 
A

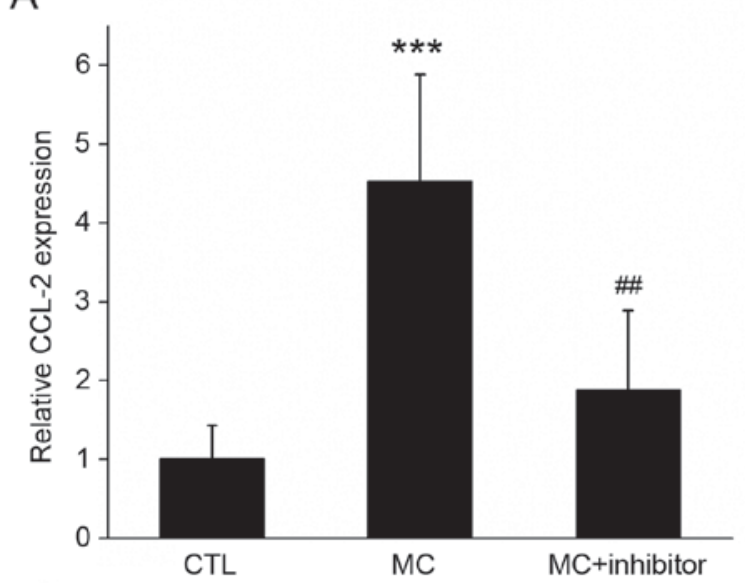

C

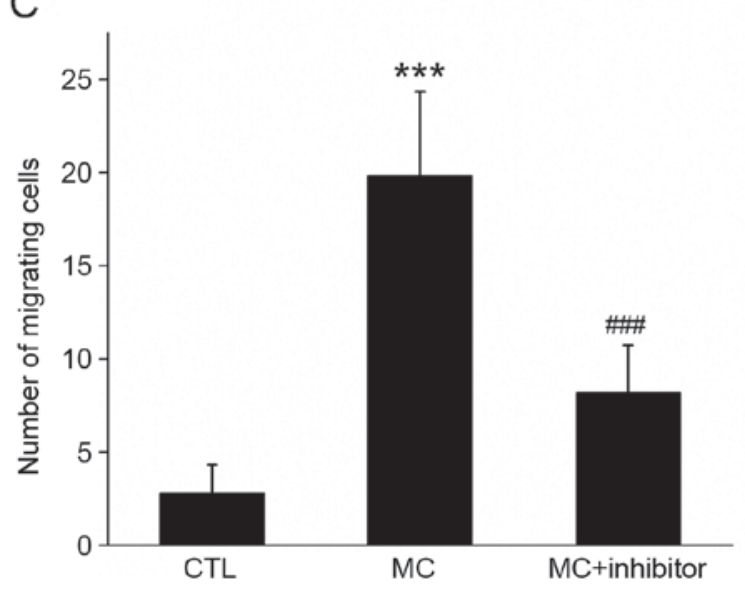

B

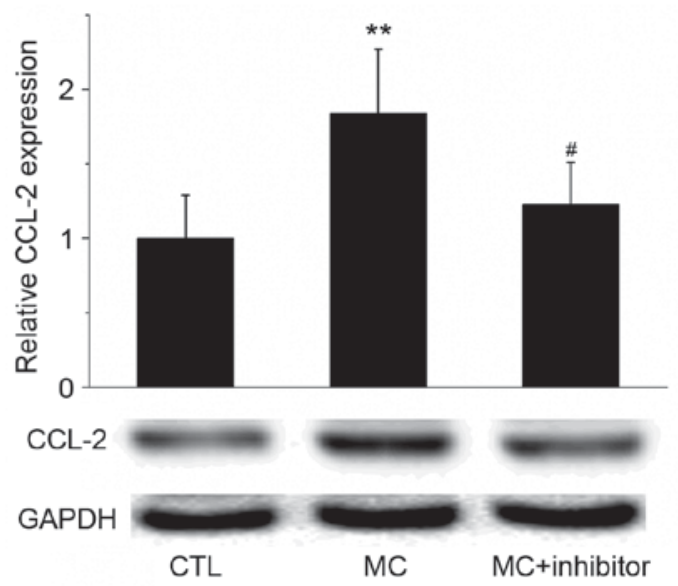

D

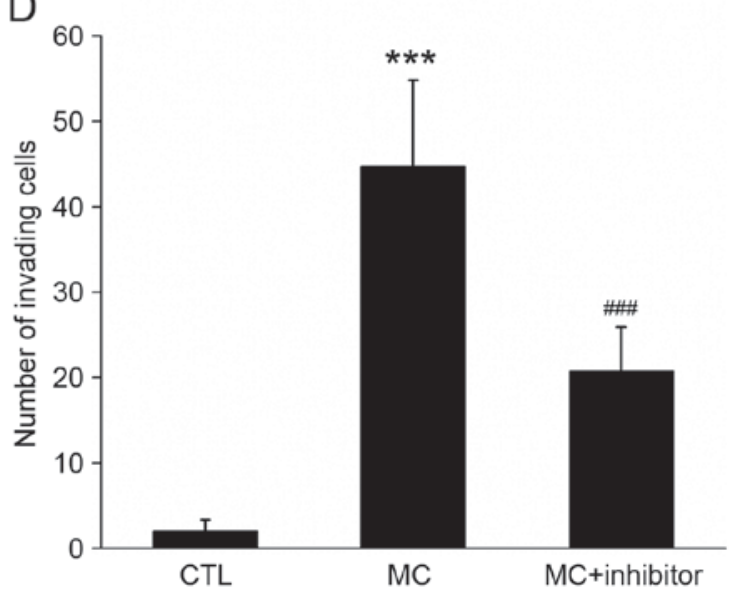

Figure 6. Effect of the inhibition of PI3K activation on co-cultured cell CCL-2 expression, invasion and migration. (A) Reverse transcription-quantitative polymerase chain reaction and (B) western blot analysis determined that, following co-culture with MC, the relative expression of CCL-2 mRNA and protein in gastric cancer cells was increased, compared with the control. However, if cells were pre-treated with the specific PI3K inhibitor wortmannin, the increase in the gene and protein expression levels of CCL-2 was significantly inhibited. GADPH was used as the loading control. (C) Migration and (D) invasion assays demonstrating that the migration of gastric cancer cells increased with MC co-culture, but decreased following the addition of the PI3K inhibitor wortmannin. ${ }^{* * *} \mathrm{P}<0.001 \mathrm{MC}$ vs. CTL; ${ }^{\#} \mathrm{P}<0.05,{ }^{\#} \mathrm{P}<0.01$ and ${ }^{\# \#} \mathrm{P}<0.001 \mathrm{MC}$ vs. MC+ inhibitor. PI3K, phosphoinositide 3-kinase; CCL-2, C-C motif chemokine ligand 2; CCR2, C-C motif chemokine receptor 2; MC, mast cell co-culture group; CTL, control group.

the intermediate-to-advanced stage, and the 5-year survival rate is low $(21,22)$. Despite resective surgery, recurrence and metastasis occur in $>50 \%$ of the patients (2). Therefore, it is necessary to explore the underlying molecular mechanisms for gastric cancer metastasis, and identify novel markers and therapeutic targets, in order to determine an improved theoretical basis for the diagnosis and treatment of gastric cancer. The underlying molecular mechanism of gastric cancer is complex; the disease course and risk factors are associated with inflammation (3). The progression of gastric cancer is regulated by the interactions of a number of types of cell in the tumor microenvironment, and the microenvironment of gastric cancer may alter the phenotype of immunoinflammatory cells (6). It is now evident that inflammatory cells have powerful effects on tumor development, and gastric cancer may be included (6).

The infiltration of mast cells was previously observed to increase in lymphoma, breast cancer and other types of tumor compared with non-tumor tissue $(9,10)$. The analysis of tissue samples from 30 patients with gastric cancer demonstrated that that the number of mast cells exhibited a positive association with the vascular density, and a negative association with the patient prognosis (23). The present study provides evidence that mast cells serve a function in gastric cancer. The infiltration ratio of mast cells in 40 gastric cancer tissues and adjacent cancer tissues were analyzed. The results demonstrated that the proportion of mast cells in gastric cancer tissues were significantly increased, compared with that in the paracancerous tissues, which was validated using immunohistochemical staining with tryptase. In addition, the Transwell migration and invasion assay results revealed that co-culture with mast cells significantly enhanced the migratory and invasive abilities of gastric cancer cells. Furthermore, the results of the CCK-8 assay suggested that co-culture with mast cells may promote the proliferation of gastric cancer cells, whereas the double fluorescence staining result suggested that co-culture inhibited the $\mathrm{H}_{2} \mathrm{O}_{2}$-induced apoptosis of gastric cancer cells.

It was identified in previous studies that the role of mast cells in different tumor types varies; mast cell infiltration has been associated with both tumor promotion and suppression. For example, in primary cutaneous lymphoma, it was demonstrated that mast cells promoted the growth of tumor cells (24). In colorectal cancer, however, the infiltration of the tumor by mast cells was negatively associated with lymph node or 
distant metastasis (25). Previous studies have suggested that interstitial mast cells in breast cancer were associated with an improved prognosis $(26,27)$. Therefore, the effect of mast cells may be associated with the specific type and stage of the tumor.

A previous study indicated that chemokines and their receptors are involved in tumor cell biology, including the regulation of cell proliferation, apoptosis, metastasis, angiogenesis and the immune response to tumors (13). CCL-2 is a key molecule in the regulation of inflammation that is secreted by monocytes, epithelial cells or certain types of tumor cell (12). CCR2, as a member of the chemokine receptor family, is associated with cell migration and other functions in tumors (28). Studies have indicated that CCL-2 or CCR2 are highly expressed in a number of types of tumor and serve functions in their development $(13,15)$. The results of RT-qPCR and western blot analysis in the present study validated that the expression of CCL-2 and CCR2 mRNA and proteins in gastric cancer tissues were significantly increased compared with adjacent tissues, consistent with a previous study (29). At present, the proposed molecular mechanisms for the promotion of tumor development by CCL-2 expression are: i) The recruitment of macrophages and influence of their phenotype, quantity and function; ii) the promotion of tumor angiogenesis; iii) effects on the regulation of the immune response; and iv) acting directly on tumor cells, e.g. stimulating tumor cells to promote stem cell characteristics and spheroiding phenotype $(30,31)$. CCL-2 may regulate the association between normal cells and tumor cells in the microenvironment, and promote the metastasis of prostate cancer (32). It was previously identified that the expression of CCL-2 in the tissue and serum of patients with oral squamous cell carcinoma was upregulated, and the tumor size of mice was significantly decreased when CCL-2 was inhibited (33). Rokavec et al (14) demonstrated that CCL-2 may directly promote the transformation of mammary epithelial cells. The results of the present study suggested that when gastric cancer cells were co-cultured with mast cells, the expression of CCL-2 mRNA and protein increased; however, when the gastric cancer cells were pre-treated with a PI3K inhibitor, wortmannin, the elevation of CCL-2 was inhibited, in addition to the effects of mast cells on gastric cancer cell proliferation, invasion and migration. This result indicated that CCL-2 may promote the migration and invasion of gastric cancer cells.

SCF is produced by stromal cells in the bone marrow microenvironment and may regulate mast cell growth through coordination with other types of cytokine, induction of the proliferation of stem/progenitor cells and the inhibition of apoptosis, and thus affect the development of mast cells (16). c-Kit is a transmembrane protein with tyrosine kinase activity; its ligand is SCF, which is expressed by a number of types of cell, including hematopoietic stem cells, mast cells, melanocytes and Cajal cells from the gastrointestinal tract (19). It was demonstrated that c-Kit is highly expressed in a number of types of malignant tumor, including mast cell leukemia, neuroblastoma, malignant melanoma, endometrial cancer, ovarian cancer and small cell lung cancer (19). Furthermore, c-Kit is used as a marker for the diagnosis of the gastrointestinal stromal tumors, and a target for therapy (19). The results of the present study indicated that the expression levels of SCF and c-Kit proteins in gastric cancer tissues were significantly increased compared the adjacent tissues. In addition, it was identified that the expression of p-Akt in gastric cancer tissues was significantly increased compared with the adjacent tissues. Based on the data of the present study, we hypothesize that the SCF/c-Kit signaling pathway may activate the PI3K-Akt signaling pathway in gastric cancer, and participate in the development and metastasis of gastric cancer. To test this, the specific PI3K inhibitor wortmannin was used; when treated with wortmannin, the extent of the promotion of gastric cancer cell migration and invasion of by mast cells was significantly inhibited. Therefore, activation of the SCF/c-Kit signaling pathway may activate PI3K-Akt and enhance the effect of mast cells by upregulating CCL-2 secretion in gastric cancer cells.

In the present study, the underlying molecular mechanisms of mast cells in gastric cancer were investigated. It was identified with flow cytometry and immunohistochemistry that the infiltration ratio of mast cells in gastric cancer tissues was significantly increased compared with the adjacent cancer tissues. Additionally, the expression level of CCL-2 and CCR2 mRNA and protein was increased significantly in gastric cancer tissues compared with adjacent tissues. Furthermore, in vitro cellular experimental results demonstrated that mast cells may promote the proliferation, migration and invasion of gastric cancer cells, and inhibit apoptosis. We therefore hypothesize that activation of the SCF/c-Kit signaling pathway may promote the expression of CCL-2 by activating PI3K-Akt to promote the occurrence and development of gastric cancer.

In conclusion, the results of the present study provide new insight on the mechanism of gastric cancer development, and may identify potential novel targets for clinical treatment.

\section{References}

1. Rugge M, Fassan M and Graham DY: Epidemiology of gastric cancer. Gastric Cancer: 23-34, 2015.

2. Ferlay J, Shin HR, Bray F, Forman D, Mathers C and Parkin DM: Estimates of worldwide burden of cancer in 2008: GLOBOCAN 2008. Int J Cancer 127: 2893-2917, 2010.

3. Trinchieri G: Cancer and inflammation: An old intuition with rapidly evolving new concepts. Annu Rev Immunol 30: 677-706, 2012.

4. Chung HW and Lim JB: Role of the tumor microenvironment in the pathogenesis of gastric carcinoma. World J Gastroenterol 20: $1667-1680,2014$.

5. Mroczko B, Łukaszewicz-Zajac M, Guzińska-Ustymowicz K, Gryko M, Czyzewska J, Kemona A, Kedra B and Szmitkowski M: Expression of matrix metalloproteinase-9 in the neoplastic and interstitial inflammatory infiltrate cells in gastric cancer. Folia Histochem Cytobiol 47: 491-496, 2009.

6. Dalgleishl AG and O'Byrne K: Inflammation and cancer. Nature 420: 1-3849, 2014.

7. Rivera LB, Meyronet D, Hervieu V, Frederick MJ, Bergsland E and Bergers G: Intratumoral myeloid cells regulate responsiveness and resistance to antiangiogenic therapy. Cell Rep 11: 577-591, 2015.

8. Theoharides TC, Alysandratos K-D, Angelidou A, Delivanis D-A, Sismanopoulos N, Zhang B, Asadi S, Vasiadi M, Weng Z, Miniati A and Kalogeromitros D: Mast cells and inflammation. Biochim Biophys Acta 1822: 21-33, 2013.

9. Raica M, Cimpean AM, Ceausu R, Ribatti D and Gaje P: Interplay between mast cells and lymphatic vessels in different molecular types of breast cancer. Anticancer Res 33: 957-963, 2013.

10. Rabenhorst A, Schlaak M, Heukamp LC, Förster A, Theurich S, von Bergwelt-Baildon M, Büttner R, Kurschat P, Mauch C, Roers A and Hartmann K: Mast cells play a protumorigenic role in primary cutaneous lymphoma. Blood 120: 2042-2054, 2012.

11. Griffith JW, Sokol CL and Luster AD: Chemokines and chemokine receptors: Positioning cells for host defense and immunity. Annu Rev Immunol 32: 659-702, 2014. 
12. Deshmane SL, Kremlev S, Amini S and Sawaya BE: Monocyte chemoattractant protein-1 (MCP-1): An overview. J Interferon Cytokine Res 29: 313-326, 2009.

13. Tsaur I, Noack A, Makarevic J, Oppermann E, Waaga-Gasser AM, Gasser M, Borgmann H, Huesch T, Gust KM, Reiter M, et al: CCL2 chemokine as a potential biomarker for prostate cancer: A pilot study. Cancer Res Treat 47: 306-312, 2014.

14. Rokavec M, Wu W and Luo JL: IL6-mediated suppression of miR-200c directs constitutive activation of inflammatory signaling circuit driving transformation and tumorigenesis. Mol Cell 45: 777-789, 2012.

15. Wu J, Liu X and Wang Y: Predictive value of preoperative serum CCL2, CCL18 and VEGF for the patients with gastric cancer. BMC Clin Pathol 13: 15, 2013.

16. Ray P, Krishnamoorthy $\mathrm{N}$ and Ray A: Emerging functions of c-kit and its ligand stem cell factor in dendritic cells: Regulators of T cell differentiation. Cell Cycle 7: 2826-2832, 2008.

17. Amagai Y, Tanaka A, Matsuda A, Jung K, Ohmori K and Matsuda H: Stem cell factor contributes to tumorigenesis of mast cells via an autocrine/paracrine mechanism. J Leukoc Biol 93 245-250, 2013.

18. Suphanantachat $\mathrm{S}$, Iwata $\mathrm{T}$, Ishihara J, Yamato $\mathrm{M}$, Okano $\mathrm{T}$ and Izumi Y: A role for c-Kit in the maintenance of undifferentiated human mesenchymal stromal cells. Biomaterials 35: 3618-3626, 2014.

19. Ashman LK and Griffith R: Therapeutic targeting of c-KIT in cancer. Expert Opin Investig Drugs 22: 103-115, 2013.

20. Huscher CG, Mingoli A, Sgarzini G, Sansonetti A, Di Paola M, Recher A and Ponzano C: Laparoscopic versus open subtotal gastrectomy for distal gastric cancer: Five-year results of a randomized prospective trial. Ann Surg 241: 232-237, 2005.

21. Chen W, Zheng R, Zhang S, Zhao P, Zeng H and Zou X: Report of cancer incidence and mortality in China, 2010. Ann Transl Med 2: 61: 2014

22. Ferro A, Peleteiro B, Malvezzi M, Bosetti C, Bertuccio P, Levi F, Negri E, La Vecchia C and Lunet N: Worldwide trends in gastric cancer mortality (1980-2011), with predictions to 2015, and incidence by subtype. Eur J Cancer 50: 1330-1344, 2014.

23. Ribatti D, Guidolin D, Marzullo A, Nico B, Annese T, Benagiano V and Crivellato E: Mast cells and angiogenesis in gastric carcinoma. Int J Exp Pathol 91: 350-356, 2010.
24. Rabenhorst A, Schlaak M, Heukamp LC, Förster A, Theurich S, von Bergwelt-Baildon M, Büttner R, Kurschat P, Mauch C, Roers A and Hartmann K: Mast cells play a protumorigenic role in primary cutaneous lymphoma. Blood 120: 2042-2054, 2012.

25. Tan SY, Fan Y, Luo HS, Shen ZX, Guo Y and Zhao LJ: Prognostic significance of cell infiltrations of immunosurveillance in colorectal cancer. World J Gastroenterol 11: 1210-1214, 2005.

26. Rajput AB, Turbin DA, Cheang MC, Voduc DK, Leung S, Gelmon KA, Gilks CB and Huntsman DG: Stromal mast cells in invasive breast cancer are a marker of favourable prognosis: A study of 4,444 cases. Breast Cancer Res Treat 107: 249-257, 2008.

27. Amini RM, Aaltonen K, Nevanlinna H, Carvalho R, Salonen L, Heikkilä P and Blomqvist C: Mast cells and eosinophils in invasive breast carcinoma. BMC Cancer 7: 165, 2007.

28. Huang Y, Chen H, Wang J, Bunjhoo H, Xiong W, Xu Y and Zhao J: Relationship between CCR2-V64I polymorphism and cancer risk: A meta-analysis. Gene 524: 54-58, 2013.

29. Tao LL, Shi SJ, Chen LB and Huang GC: Expression of monocyte chemotactic protein-1/CCL2 in gastric cancer and its relationship with tumor hypoxia. World J Gastroenterol 20: 4421-4427, 2014.

30. Qian BZ, Li J, Zhang H, Kitamura T, Zhang J, Campion LR, Kaiser EA, Snyder LA and Pollard JW: CCL2 recruits inflammatory monocytes to facilitate breast tumor metastasis. Nature 475: 222-225, 2011.

31. Low-Marchelli JM, Ardi VC, Vizcarra EA, van Rooijen N, Quigley JP and Yang J: Twist1 induces CCL2 and recruits macrophages to promote angiogenesis. Cancer Res 73: 662-671, 2013.

32. Zhang J, Patel L and Pienta KJ: CC chemokine ligand 2 (CCL2) promotes prostate cancer tumorigenesis and metastasis. Cytokine Growth Factor Rev 21: 41-48, 2010.

33. Li X, Xu Q, Wu Y, Li J, Tang D, Han L and Fan Q: A CCL2/ROS autoregulation loop is critical for cancer associated fibroblasts-enhanced tumor growth of oral squamous cell carcinoma. Carcinogenesis 35: 1362-1370, 2014.

(i) $\odot$ This work is licensed under a Creative Commons Attribution-NonCommercial-NoDerivatives 4.0 International (CC BY-NC-ND 4.0) License. 